\title{
Local Control of the Curves Using Rational Cubic Spline
}

\author{
Samsul Ariffin Abdul Karim ${ }^{1}$ and Kong Voon Pang ${ }^{2}$ \\ ${ }^{1}$ Department of Fundamental and Applied Sciences, Universiti Teknologi PETRONAS, Bandar Seri Iskandar, \\ 31750 Tronoh, Perak Darul Ridzuan, Malaysia \\ ${ }^{2}$ School of Mathematical Sciences, Universiti Sains Malaysia (USM), 11800 Minden, Penang, Malaysia \\ Correspondence should be addressed to Samsul Ariffin Abdul Karim; samsul_ariffin@petronas.com.my
}

Received 22 October 2013; Accepted 7 January 2014; Published 2 March 2014

Academic Editor: Jingxin Zhang

Copyright (C) 2014 S. A. Abdul Karim and K. Voon Pang. This is an open access article distributed under the Creative Commons Attribution License, which permits unrestricted use, distribution, and reproduction in any medium, provided the original work is properly cited.

\begin{abstract}
This paper discussed the local control of interpolating function by using rational cubic spline (cubic/quadratic) with three parameters originally proposed by the authors. The rational spline has $C^{1}$ continuity. The bounded properties of the rational cubic interpolants and shape controls of the rational interpolants are discussed in detail. The value control, inflection point control, and convexity control at a point by using the proposed rational cubic spline are constructed. Several numerical results are presented to show the capability of the method. Numerical comparisons with the existing scheme are also further elaborated. From the results, it was indicated that the scheme works well and it is comparable with the established existing scheme.
\end{abstract}

\section{Introduction}

Shape preserving is important for the visualization of the given data sets. For example, if the design requires the interpolating curves or surfaces to retain its geometric properties such as convexity, monotonicity, and positivity, any proposed method must be able to fulfill these important criteria. For example, if the data is convex, then the resultant interpolating function must be convex on entire given interval. Common spline polynomial interpolations have a unique interpolation function [1-7]. If the design requires the modification of the shape of the interpolating curves, the data need to be changed. Thus those mentioned methods are not capable of changing the shape of the curves with the same data points (interpolation points). To meet the needs of industrial expertise, the interpolating function should be able to modify the shape of the interpolating curves by either locally or globally altering the values of its shape parameters. Motivated by this, many researchers have developed many rational interpolants with shape parameters for shape preserving interpolation. For instance, Sarfraz [8], Sarfraz et al. [9], Hussain et al. [10], Shaikh et al. [11], and Tian et al. [12] have proposed different types of rational spline interpolants (cubic/cubic or cubic/quadratic form) for the shape control. The main idea is that the sufficient condition on the shape parameters for the monotonicity, convexity, or positivity preserving will be derived while the other parameter(s) will be let as free parameter(s) and we may have many types of interpolating curves with different values of respective shape parameter.

Besides the use of rational spline for shape preserving, there exist many research papers discussing the local control of interpolating curves. Strictly speaking, the rational splines were used to meet the design requirement such as point control, inflection-point control, or convexity control of interpolating function. Bao et al. [13] have discussed the point control of the interpolating function of the form cubic/quadratic with two parameters. Meanwhile Bao et al. [14] have proposed a new local method for controlling the shape of interpolating curves by using rational cubic spline of the form cubic/cubic. Duan et al. [15] have discussed the local control of interpolating function by utilizing the rational cubic spline of the form cubic/linear and Bao et al. [16] have explored the use of blending interpolator by using the weighted between the rational cubic spline (cubic/linear) and cubic Hermite spline polynomial. These ideas have been extended by Sun et al. [17]. They apply their blending interpolator for value control with minimal strain energy meanwhile Duan et al. [18] have used rational cubic spline 
(cubic/quadratic) of Gregory et al. [19] to control the value, convexity, and inflection-point control of the interpolation. Due to the fact that there are some cases where there are no parameters values that can be used in order to control the final shape of the curves, Bao et al. [16] and Sun et al. [17] have proposed blending interpolator that will enable the local control of the interpolating curves. Pan and Sun [20] have extended the rational cubic spline interpolant of Bao et al. [13] (cubic/quadratic) with one parameter for local control. Even though many researchers have published many rational splines for the local control of the interpolating curve, there is still one question that is worth asking and may be useful to be considered. If the design requires that the value of the interpolating function at the point $t^{*}, P\left(t^{*}\right)$ is equal to real number $N$, that is, $P\left(t^{*}\right)=N$, the main question is, how to select or choose the value of $N$ ? In Duan et al. [18] the authors did not mention how to ensure that the user will choose the right $N$. Otherwise the user may need to keep changing the values of each shape parameter that satisfies the sufficient condition for the value-point control of the interpolating function. Motivated by this question, in this paper, the authors will discuss the local control (point, convex, and inflection point) by using the recent rational cubic spline originally proposed by Karim and Kong [21-23]. These rational cubic splines (cubic/quadratic) have three parameters. The main scientific contributions of this paper are summarized as follows.

(i) In this paper a new rational cubic spline (cubic/ quadratic) with the three parameters initiated by Karim and Kong [21-23] has been used for local control of the interpolation data.

(ii) Our methods have three parameters while in the work of Duan et al. [18] they have only two parameters. By having three parameters, the local control of the interpolating function can be achieved with a greater flexibility. The suitable parameter value can be obtained by simply solving linear equation. This can be seen clearly in the numerical examples later.

(iii) Our methods are based on function values and the derivative is given and it is different from the works of Bao et al. [13], Bao et al. [14], Duan et al. [15], and Sun et al. [17] in which they rely on true function values and no derivative is given.

(iv) Our method works well with the tested data sets and it is comparable with the works of Duan et al. [18].

(v) Our rational scheme is based on spline function while in Bashir and Alim [24] the interpolant is based on trigonometric spline.

The remainder of the paper is organized as follows. Section 2 introduces the rational cubic spline with the three parameters originally initiated by Karim and Kong [21]. Section 3 discusses the bounded property of the rational interpolation. The value control of the interpolating function is discussed in Section 4 with numerical examples. Section 5 is devoted to the inflection-point and convexity control of the interpolating curves with numerical examples. This section also includes the comparison between the proposed local control and the work of Duan et al. [18]. A summary and conclusions are given in Section 6.

\section{Rational Cubic Spline Interpolant}

This section will introduce the rational cubic spline interpolant with the three parameters originally proposed by Karim and Kong [21]. Let us assume that $\left(t_{i}, f_{i}, d_{i}\right), i=$ $0,1, \ldots, n$, is a given set of data where $t_{0}<t_{1}<\cdots<t_{n}$. Let $h_{i}=t_{i+1}-t_{i}, \Delta_{i}=\left(f_{i+1}-f_{i}\right) / h_{i}$, and a local variable $\theta=\left(t-t_{i}\right) / h_{i}$ where $0 \leq \theta \leq 1$.

For $t \in\left[t_{i}, t_{i+1}\right], i=0,1,2, \ldots, n-1$,

$$
\left.P(t)\right|_{\left[t_{i}, t_{i+1}\right]}=\frac{p_{i}(t)}{q_{i}(t)}, \quad i=0,1, \ldots, n-1,
$$

where

$$
\begin{gathered}
p_{i}(t)=A_{0}(1-\theta)^{3}+A_{1} \theta(1-\theta)^{2}+A_{2} \theta^{2}(1-\theta)+A_{3} \theta^{3}, \\
q_{i}(t)=(1-\theta)^{2} \alpha_{i}+\theta(1-\theta)\left(2 \alpha_{i} \beta_{i}+\gamma_{i}\right)+\theta^{2} \beta_{i},
\end{gathered}
$$

with

$$
\begin{gathered}
A_{0}=\alpha_{i} f_{i}, \quad A_{1}=\left(2 \alpha_{i} \beta_{i}+\alpha_{i}+\gamma_{i}\right) f_{i}+\alpha_{i} h_{i} d_{i}, \\
A_{2}=\left(2 \alpha_{i} \beta_{i}+\beta_{i}+\gamma_{i}\right) f_{i+1}-\beta_{i} h_{i} d_{i+1}, \quad A_{3}=\beta_{i} f_{i+1} .
\end{gathered}
$$

The parameters $\alpha_{i}, \beta_{i}>0$, and $\gamma_{i} \geq 0$. When $\alpha_{i}=\beta_{i}=1$, $\gamma_{i}=0$, the rational cubic interpolant in (1) is a standard cubic Hermite spline given as follows:

$$
\begin{aligned}
P(t)= & (1-\theta)^{2}(1+2 \theta) f_{i}+\theta^{2}(3-2 \theta) f_{i+1} \\
& +\theta(1-\theta)^{2} d_{i}-\theta^{2}(1-\theta) d_{i+1} .
\end{aligned}
$$

The shapes parameters $\alpha_{i}$ and $\beta_{i}, i=0,1,2, \ldots, n-1$, are free parameters (independent) while the shape preserving (positivity, monotonicity, and convexity) as well as data constrained modeling are derived from the other parameter $\gamma_{i}$ (dependent). The two parameters $\alpha_{i}, \beta_{i}$ can be used to refine and modify the final shape of the interpolating curve $[21,24]$.

\section{The Bounded Property of the Interpolation}

As mentioned by Duan et al. [18], in order to study the bounded property of the interpolation, without loss of generality, the interpolation in a subinterval $\left[t_{i}, t_{i+1}\right], i=$ $0,1,2, \ldots, n-1$, will be considered. The interpolating function $P(t)$ in (1) can be rewritten as follows:

$$
\begin{aligned}
P(t)= & \omega_{0}\left(\theta, \alpha_{i}, \beta_{i}, \gamma_{i}\right) f_{i}+\omega_{1}\left(\theta, \alpha_{i}, \beta_{i}, \gamma_{i}\right) f_{i+1} \\
& +\omega_{2}\left(\theta, \alpha_{i}, \beta_{i}, \gamma_{i}\right) h_{i} d_{i}+\omega_{3}\left(\theta, \alpha_{i}, \beta_{i}, \gamma_{i}\right) h_{i} d_{i+1}
\end{aligned}
$$

where

$$
\begin{aligned}
& \omega_{0}\left(\theta, \alpha_{i}, \beta_{i}, \gamma_{i}\right) \\
& \quad=\frac{(1-\theta)^{3} \alpha_{i}+\theta(1-\theta)^{2}\left(2 \alpha_{i} \beta_{i}+\alpha_{i}+\gamma_{i}\right)}{(1-\theta)^{2} \alpha_{i}+\theta(1-\theta)\left(2 \alpha_{i} \beta_{i}+\gamma_{i}\right)+\theta^{2} \beta_{i}},
\end{aligned}
$$




$$
\begin{aligned}
& \omega_{1}\left(\theta, \alpha_{i}, \beta_{i}, \gamma_{i}\right) \\
& =\frac{\theta^{3} \beta_{i}+\theta^{2}(1-\theta)\left(2 \alpha_{i} \beta_{i}+\beta_{i}+\gamma_{i}\right)}{(1-\theta)^{2} \alpha_{i}+\theta(1-\theta)\left(2 \alpha_{i} \beta_{i}+\gamma_{i}\right)+\theta^{2} \beta_{i}}, \\
& \omega_{2}\left(\theta, \alpha_{i}, \beta_{i}, \gamma_{i}\right) \\
& =\frac{\theta(1-\theta)^{2} \alpha_{i}}{(1-\theta)^{2} \alpha_{i}+\theta(1-\theta)\left(2 \alpha_{i} \beta_{i}+\gamma_{i}\right)+\theta^{2} \beta_{i}}, \\
& \omega_{3}\left(\theta, \alpha_{i}, \beta_{i}, \gamma_{i}\right) \\
& =-\frac{\theta^{2}(1-\theta) \beta_{i}}{(1-\theta)^{2} \alpha_{i}+\theta(1-\theta)\left(2 \alpha_{i} \beta_{i}+\gamma_{i}\right)+\theta^{2} \beta_{i}} .
\end{aligned}
$$

The rational functions $\omega_{j}\left(\theta, \alpha_{i}, \beta_{i}, \gamma_{i}\right), j=0,1,2,3$, are called interpolating bases of $P(t)$. Now, for $\theta \in(0,1)$ Property 1 holds.

Property 1. The interpolation bases have the following properties.

(a) $\omega_{0}\left(\theta, \alpha_{i}, \beta_{i}, \gamma_{i}\right)+\omega_{1}\left(\theta, \alpha_{i}, \beta_{i}, \gamma_{i}\right)=1$.

(b) $\omega_{2}\left(\theta, \alpha_{i}, \beta_{i}, \gamma_{i}\right)-\omega_{3}\left(\theta, \alpha_{i}, \beta_{i}, \gamma_{i}\right)=\theta(1-\theta)\left((1-\theta) \alpha_{i}+\right.$ $\left.\theta \beta_{i}\right) /\left((1-\theta)^{2} \alpha_{i}+\theta(1-\theta)\left(2 \alpha_{i} \beta_{i}+\gamma_{i}\right)+\theta^{2} \beta_{i}\right)$.

(c) $\omega_{0}\left(\theta, \alpha_{i}, \beta_{i}, \gamma_{i}\right) \geq 0, \omega_{1}\left(\theta, \alpha_{i}, \beta_{i}, \gamma_{i}\right) \geq 0$, $\omega_{2}\left(\theta, \alpha_{i}, \beta_{i}, \gamma_{i}\right) \geq 0, \omega_{3}\left(\theta, \alpha_{i}, \beta_{i}, \gamma_{i}\right) \leq 0$.

Now, $\omega_{0}\left(\theta, \alpha_{i}, \beta_{i}, \gamma_{i}\right)$ and $\omega_{1}\left(\theta, \alpha_{i}, \beta_{i}, \gamma_{i}\right)$ can be rewritten as follows:

$$
\begin{aligned}
& \omega_{0}\left(\theta, \alpha_{i}, \beta_{i}, \gamma_{i}\right) \\
& =\frac{(1-\theta)^{2} \alpha_{i}+\theta(1-\theta)^{2}\left(2 \alpha_{i} \beta_{i}+\gamma_{i}\right)}{(1-\theta)^{2} \alpha_{i}+\theta(1-\theta)\left(2 \alpha_{i} \beta_{i}+\gamma_{i}\right)+\theta^{2} \beta_{i}}, \\
& \omega_{1}\left(\theta, \alpha_{i}, \beta_{i}, \gamma_{i}\right) \\
& =\frac{\theta^{2} \beta_{i}+\theta^{2}(1-\theta)\left(2 \alpha_{i} \beta_{i}+\gamma_{i}\right)}{(1-\theta)^{2} \alpha_{i}+\theta(1-\theta)\left(2 \alpha_{i} \beta_{i}+\gamma_{i}\right)+\theta^{2} \beta_{i}} .
\end{aligned}
$$

The following theorem provides the bounded property of the interpolation function $P(t)$.

Theorem 1. Let $P(t)$ be the interpolating function defined in (1); whatever the positive values of the shape parameters $\alpha_{i}, \beta_{i}$, and $\gamma_{i}$ might be, the values of the interpolating function $P(t)$ in $\left[t_{i}, t_{i+1}\right], i=0,1,2, \ldots, n-1$, satisfy the following condition:

$$
|P(t)| \leq M+h_{i} D,
$$

where

$$
M=\max _{j=i}^{i+1}\left|f_{j}\right|, \quad D=\max _{t \in\left[t_{i}, t_{i+1}\right]}\left|f^{\prime}(t)\right| .
$$

Proof. Due to the locality of the interpolation function, we only consider the function $P(t)$ in subinterval $\left[t_{i}, t_{i+1}\right], i=$
$0,1,2, \ldots, n-1$. Now, from basic calculus and (5), the following expression can be obtained:

$$
\begin{aligned}
|P(t)| \leq & \left|\omega_{0}\left(\theta, \alpha_{i}, \beta_{i}, \gamma_{i}\right) f_{i}\right|+\left|\omega_{1}\left(\theta, \alpha_{i}, \beta_{i}, \gamma_{i}\right) f_{i+1}\right| \\
& +\left|\omega_{2}\left(\theta, \alpha_{i}, \beta_{i}, \gamma_{i}\right) h_{i} d_{i}\right|+\left|\omega_{3}\left(\theta, \alpha_{i}, \beta_{i}, \gamma_{i}\right) h_{i} d_{i+1}\right| .
\end{aligned}
$$

Equation (10) can be simplified to the following:

$|P(t)|$

$$
\leq M+\frac{\theta(1-\theta)\left((1-\theta) \alpha_{i}+\theta \beta_{i}\right)}{(1-\theta)^{2} \alpha_{i}+\theta(1-\theta)\left(2 \alpha_{i} \beta_{i}+\gamma_{i}\right)+\theta^{2} \beta_{i}} h_{i} D .
$$

Let $g(\theta)=\left(\theta(1-\theta)\left((1-\theta) \alpha_{i}+\theta \beta_{i}\right)\right) /\left((1-\theta)^{2} \alpha_{i}+\theta(1-\right.$ $\left.\theta)\left(2 \alpha_{i} \beta_{i}+\gamma_{i}\right)+\theta^{2} \beta_{i}\right)$. Now, $g(\theta)$ can be further simplified to the following:

$$
\begin{aligned}
g(\theta) & =\frac{\theta(1-\theta)\left((1-\theta) \alpha_{i}+\theta \beta_{i}\right)}{(1-\theta)^{2} \alpha_{i}+\theta(1-\theta)\left(2 \alpha_{i} \beta_{i}+\gamma_{i}\right)+\theta^{2} \beta_{i}} \\
& \leq \frac{\theta(1-\theta)\left((1-\theta) \alpha_{i}+\theta \beta_{i}\right)}{(1-\theta)^{2} \alpha_{i}+\theta^{2} \beta_{i}} \\
& =\frac{\theta(1-\theta)(1-\theta) \alpha_{i}}{(1-\theta)^{2} \alpha_{i}+\theta^{2} \beta_{i}}+\frac{\theta(1-\theta) \theta \beta_{i}}{(1-\theta)^{2} \alpha_{i}+\theta^{2} \beta_{i}} \\
& \leq \frac{\theta(1-\theta)(1-\theta) \alpha_{i}}{(1-\theta)^{2} \alpha_{i}}+\frac{\theta(1-\theta) \theta \beta_{i}}{+\theta^{2} \beta_{i}} \\
& =\theta+1-\theta=1 .
\end{aligned}
$$

The results in (8) are obtained from (10) and (11). This completes the proof of Theorem 1 .

Remark 2. The bounded property given by (10) is the same as the bounded property of the rational cubic spline by Duan et al. [18].

\section{Value Control of the Interpolation}

It is a common fact that the shape of the interpolation curves depends on the given interpolation data. By having parameters in the description of the rational interpolant defined in (1), the final shape of the interpolation curves can be changed by altering the values of each shape parameter without the need to change the interpolation data itself. Thus, the user may manipulate the values of each of the parameters until the desired interpolating curves are obtained. Karim and Kong [21] have applied the rational spline defined in (1) for positivity preserving by finding the suitable values of one shape parameter while the other two, $\alpha_{i}$ and $\beta_{i}$, are free parameters. Motivated by this finding, in this section this rational cubic spline of Karim and Kong [21] defined by (1) will be used for point-value control for interpolation data.

In what follows, we adopted the ideas from Duan et al. [18]. It started with the following problem statement. 
If the practical design requires the value of the interpolating function $P(t)$ defined in (1) at the point $t^{*}$ (let $\theta^{*}$ be its local coordinate) to be equal to a real number $N$, that is, $P\left(t^{*}\right)=N$, where $N$ is between $f_{i}$ and $f_{i+1}$, what parameters values ensuring the resulting interpolating function will satisfy this requirement?

The above problem statement can be rewritten as follows:

$$
\begin{aligned}
N= & \omega_{0}\left(\theta^{*}, \alpha_{i}, \beta_{i}, \gamma_{i}\right) f_{i}+\omega_{1}\left(\theta^{*}, \alpha_{i}, \beta_{i}, \gamma_{i}\right) f_{i+1} \\
& +\omega_{2}\left(\theta^{*}, \alpha_{i}, \beta_{i}, \gamma_{i}\right) h_{i} d_{i}+\omega_{3}\left(\theta^{*}, \alpha_{i}, \beta_{i}, \gamma_{i}\right) h_{i} d_{i+1} .
\end{aligned}
$$

After some simplification, (13) is equivalent to the following:

$$
a \alpha_{i}+b \beta_{i}+c \rho_{i}=0
$$

where

$$
\begin{gathered}
a=\left(1-\theta^{*}\right)^{2}\left[N-f_{i}-h_{i} d_{i} \theta^{*}\right], \\
b=\theta^{* 2}\left[N-f_{i+1}+h_{i} d_{i+1}\left(1-\theta^{*}\right)\right], \\
c=\left(1-\theta^{*}\right) \theta^{*}\left[N-f_{i}\left(1-\theta^{*}\right)-f_{i+1} \theta^{*}\right], \\
\rho_{i}=2 \alpha_{i} \beta_{i}+\gamma_{i} .
\end{gathered}
$$

If there exist positive parameters $\alpha_{i}, \beta_{i}$, and $\gamma_{i}$ that satisfy (14), (13) holds. Thus we have the following theorem.

Theorem 3. Given the interpolating data $\left(t_{i}, f_{i}, d_{i}\right), i=$ $0,1, \ldots, n$ and the interpolating function $P(t)$ defined by (1). For each $t \in\left[t_{i}, t_{i+1}\right], i=0,1,2, \ldots, n-1$, the sufficient condition for the existing positive parameters $\alpha_{i}, \beta_{i}$, and $\gamma_{i}$ to satisfy $P\left(t^{*}\right)=N$ is that the number of the sign changes of the sequence $\{a, b, c\}$ is not equal to zero.

Example 4. Due to the fact that the interpolation is local and without loss of generality, it is enough to just consider the interpolation in $[0,1]$. We test the value control by using the interpolation data from Duan et al. [18].

In the interpolation interval $[0,1]$, it is clear that $\theta=t$. Before we can proceed to control the shape of the interpolating curves, we need to consider that, in the sufficient condition given in (14), there are terms involving $2 \alpha_{i} \beta_{i}$ and $\gamma_{i}$. Thus for the simplicity and to ensure the point-value control can be achieved without any difficulties, and to solve the equation given in (14), we need to input two parameters' value. It can be that the given parameters are either $\alpha_{i}$ and $\beta_{i}$ or $\alpha_{i}$ and $\gamma_{i}$ or $\beta_{i}$ and $\gamma_{i}$, respectively. The remaining parameter will be calculated from (14).

Let $\alpha_{i}=2, \beta_{i}=1$, and $\gamma_{i}=1$; the interpolating function $P(t)$ in the interval $[0,1]$ is given by

$$
\begin{aligned}
P_{1}(t) & =\frac{6(1-t)^{3}+19(1-t)^{2} t+34(1-t) t^{2}+6 t^{3}}{2(1-t)^{2}+5 t(1-t)+t^{2}} \\
& =\frac{6+t+14 t^{2}-15 t^{3}}{2+t-2 t^{2}} .
\end{aligned}
$$

Figure 1 shows the graph of the rational interpolation curve $P_{1}(t)$ with the parameters $\alpha_{i}=2, \beta_{i}=1$, and $\gamma_{i}=1$.

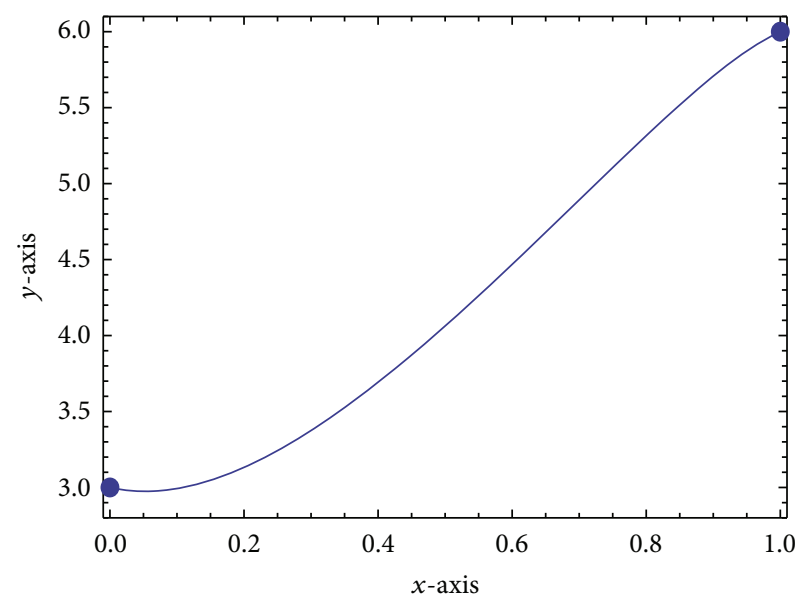

Figure 1: The graph of the interpolating curve $P_{1}(t)$ with the parameters $\alpha_{i}=2, \beta_{i}=1$, and $\gamma_{i}=1$.

Now from (1), $P_{1}(0.5)=65 / 16=4.0625$. If the design requires $P_{1}(0.5)=4$, then (14) becomes

$$
3 \alpha_{i}-2 \beta_{i}-\left(2 \alpha_{i} \beta_{i}+\gamma_{i}\right)=0
$$

Selecting with the parameters $\alpha_{i}=1.5, \beta_{i}=0.5$, and $\gamma_{i}=2$ which satisfy (14), then the interpolating function $P(t)$ in the interval $[0,1]$ becomes

$$
\begin{aligned}
P_{2}(t) & =\frac{4.5(1-t)^{3}+13.5(1-t)^{2} t+23(1-t) t^{2}+3 t^{3}}{1.5(1-t)^{2}+3.5 t(1-t)+0.5 t^{2}} \\
& =\frac{4.5+9.5 t^{2}-11 t^{3}}{1.5+0.5 t-1.5 t^{2}} .
\end{aligned}
$$

Figure 2 shows the graph of the rational interpolation curve $P_{2}(t)$ with the parameters $\alpha_{i}=1.5, \beta_{i}=0.5$, and $\gamma_{i}=2$. Simple calculation verified that $P_{2}(0.5)=4$.

Other possible choices of parameters $\alpha_{i}, \beta_{i}$, and $\gamma_{i}$ are $\alpha_{i}=$ $2, \beta_{i}=0.5$, and $\gamma_{i}=3$. These choices also satisfy (14). Now the interpolating function $P(t)$ in $[0,1]$ becomes

$$
\begin{aligned}
P_{3}(t) & =\frac{6(1-t)^{3}+19(1-t)^{2} t+32(1-t) t^{2}+3 t^{3}}{2(1-t)^{2}+5 t(1-t)+0.5 t^{2}} \\
& =\frac{6+t+12 t^{2}-16 t^{3}}{2+t-2.5 t^{2}} .
\end{aligned}
$$

Figure 3 shows the graph of the rational interpolation curve $P_{2}(t)$ with the parameters $\alpha_{i}=2, \beta_{i}=0.5$, and $\gamma_{i}=3$. Simple calculation verified that $P_{3}(0.5)=4$.

Figure 4 shows the combination of the graphs in Figures 2 and 3.

It can be seen clearly that both interpolation functions $P_{2}(t)$ and $P_{3}(t)$ are almost similar. These show that no matter what the positive value of $\alpha_{i}, \beta_{i}$, and $\gamma_{i}$ might be, as long as they satisfy (14), the resulting interpolating functions are almost similar to each other. Figure 5 shows the graph of interpolating function of $P_{1}(t)$ (blue) and $P_{2}(t)$ (red). 


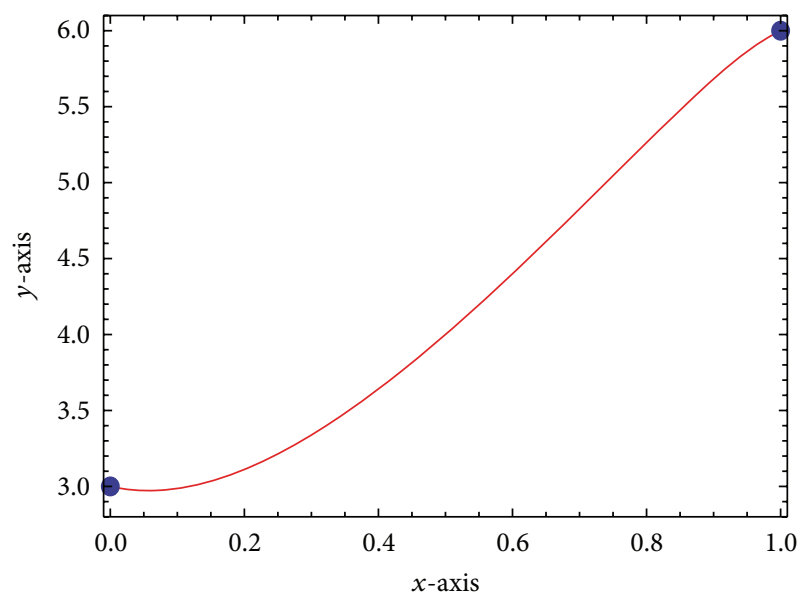

Figure 2: The graph of the interpolating curve $P_{2}(t)$ with the parameters $\alpha_{i}=1.5, \beta_{i}=0.5$, and $\gamma_{i}=2$.

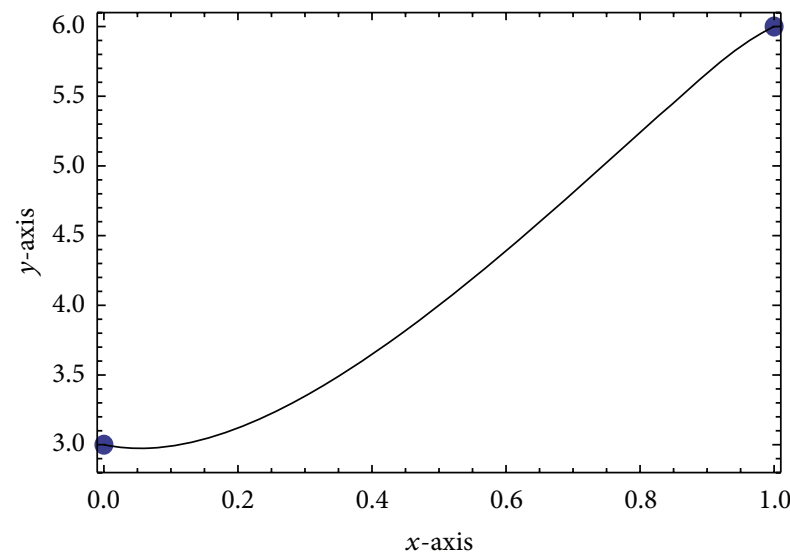

Figure 3: The graph of the rational interpolation curve $P_{2}(t)$ with the parameters $\alpha_{i}=2, \beta_{i}=0.5$, and $\gamma_{i}=3$.

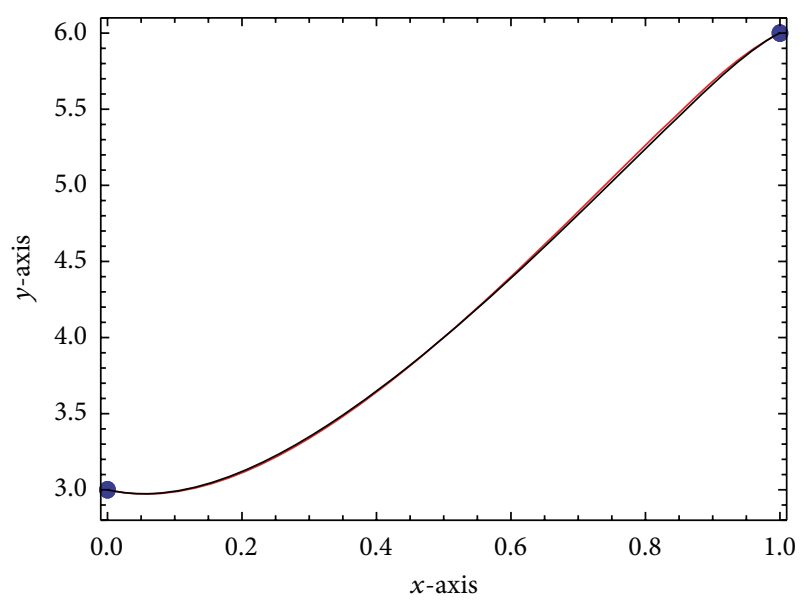

Figure 4: The graph of the rational interpolation curves $P_{2}(t)$ (red) and $P_{3}(t)$ (black).

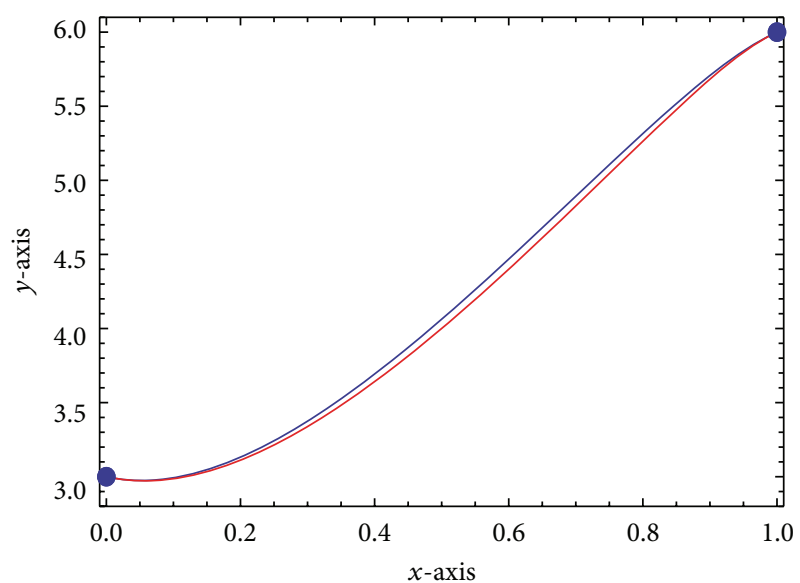

FIGURE 5: The graph of the rational interpolation curves $P_{1}(t)$ (blue) and $P_{2}(t)(\mathrm{red})$.

Now if the design requires $P_{4}(0.5)=3.8$ then (14) becomes

$$
13 \alpha_{i}-12 \beta_{i}-7\left(2 \alpha_{i} \beta_{i}+\gamma_{i}\right)=0 .
$$

Selecting with the parameters $\alpha_{i}=1.5, \beta_{i}=0.5$, and $\gamma_{i}=6 / 7$ which satisfy (14), then the interpolating function $P(t)$ in the interval $[0,1]$ becomes

$$
\begin{aligned}
& P_{4}(t) \\
& =\frac{6(1-t)^{3}+(88 / 7)(1-t)^{2} t+(134 / 7)(1-t) t^{2}+3 t^{3}}{2(1-t)^{2}+(20 / 7) t(1-t)+(1 / 2) t^{2}} \\
& =\frac{2\left(-42+38 t-84 t^{2}+67 t^{3}\right)}{-28+16 t+5 t^{2}} .
\end{aligned}
$$

Figure 6 shows the graph of the rational interpolation curve $P_{4}(t)$ with the parameters $\alpha_{i}=1.5, \beta_{i}=0.5$, and $\gamma_{i}=6 / 7$. Simple calculation verified that $P_{3}(0.5)=3.8$.

Now if the design requires $P_{5}(0.5)=4.2$, then (14) becomes

$$
17 \alpha_{i}-8 \beta_{i}-3\left(2 \alpha_{i} \beta_{i}+\gamma_{i}\right)=0 .
$$

Selecting with the parameters $\alpha_{i}=1.5, \beta_{i}=1$, and $\gamma_{i}=17 / 6$ which satisfy (14), then the interpolating function $P(t)$ in the interval $[0,1]$ becomes

$$
\begin{aligned}
P_{5}(t) & \\
& =\frac{(9 / 2)(1-t)^{3}+(41 / 2)(1-t)^{2} t+39(1-t) t^{2}+6 t^{3}}{(3 / 2)(1-t)^{2}+(35 / 6) t(1-t)+t^{2}} \\
& =\frac{27+42 t+69 t^{2}-102 t^{3}}{9+17 t-20 t^{2}} .
\end{aligned}
$$

Figure 7 shows the graph of the rational interpolation curve $P_{5}(t)$ with the parameters $\alpha_{i}=1.5, \beta_{i}=1$, and $\gamma_{i}=17 / 6$. Simple calculation verified that $P_{5}(0.5)=4.2$. 


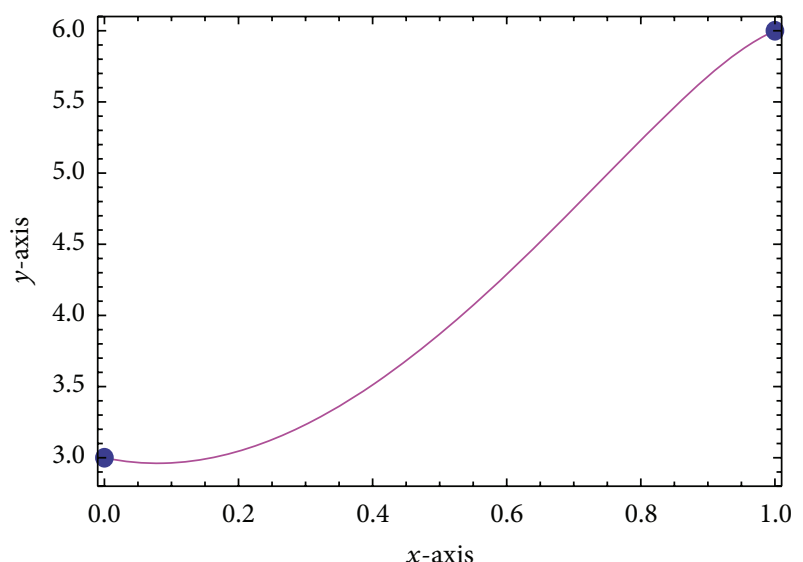

FIgURE 6: The graph of the rational interpolation curve $P_{4}(t)$ with the parameters $\alpha_{i}=1.5, \beta_{i}=0.5$, and $\gamma_{i}=6 / 7$.

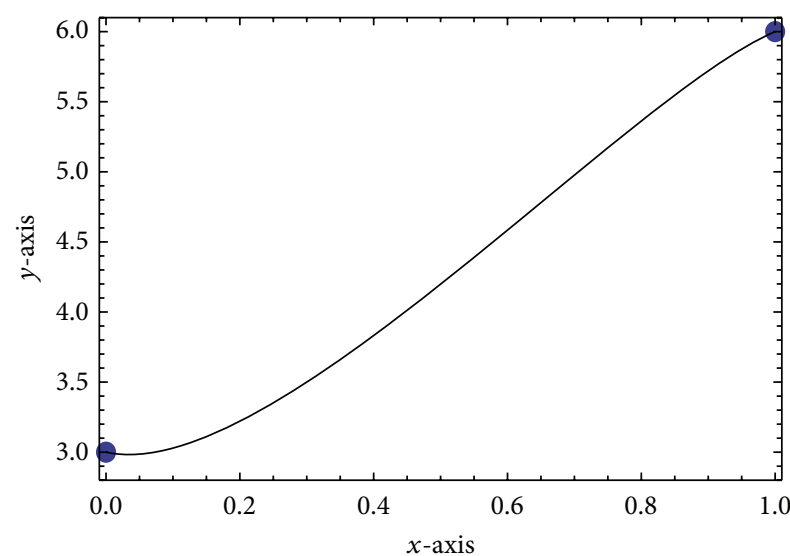

FIGURE 7: The graph of the rational interpolation curve $P_{5}(t)$ with the parameters $\alpha_{i}=1.5, \beta_{i}=1$, and $\gamma_{i}=17 / 6$.

Figure 8 shows the graph of the rational interpolation curves $P_{1}(t)$ (blue), $P_{4}(t)$ (purple), and $P_{5}(t)$ (gray), respectively, and Figure 9 shows the graph of the rational interpolation curves $P_{1}(t)$ (blue), $P_{2}(t)$ (red), $P_{4}(t)$ (purple), and $P_{5}(t)$ (gray), respectively.

Now we arrive at the main objective of this paper. From the sufficient condition in (14), if the users have chosen any suitable value of $N$, then we can obtain the parameters $\alpha_{i}, \beta_{i}$, and $\gamma_{i}$ by solving (14). To solve the equation, in this paper two parameters have been preassigned and the remaining parameter is calculated from the solution in (14). But one question still remains to be answered. What the value of $N$ might be? To answer this question, we begin with the following assumption. If the interpolating function at $t=1 / 2$ has value $N$, that is $P(1 / 2)=N$ (the value of $t$ can be any positive value in $(0,1))$, then we have the following:

$$
P\left(\frac{1}{2}, \alpha_{i}, \beta_{i}, \gamma_{i}\right)=\frac{5 \alpha_{i}+10 \beta_{i}+9 \gamma_{i}+18 \alpha_{i} \beta_{i}}{2\left(\alpha_{i}+\beta_{i}+\gamma_{i}+2 \alpha_{i} \beta_{i}\right)} .
$$

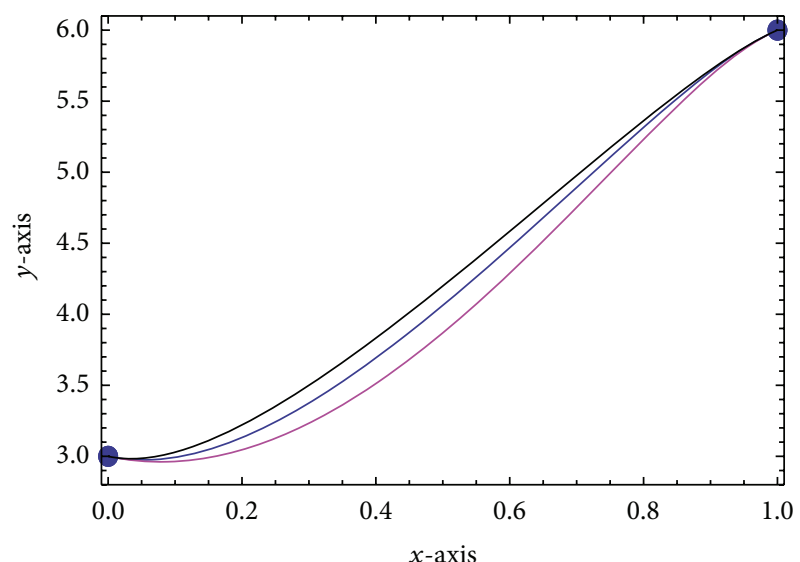

FIGURE 8: The graph of the rational interpolation curves $P_{1}(t)$ (blue), $P_{4}(t)$ (purple), and $P_{5}(t)$ (gray).

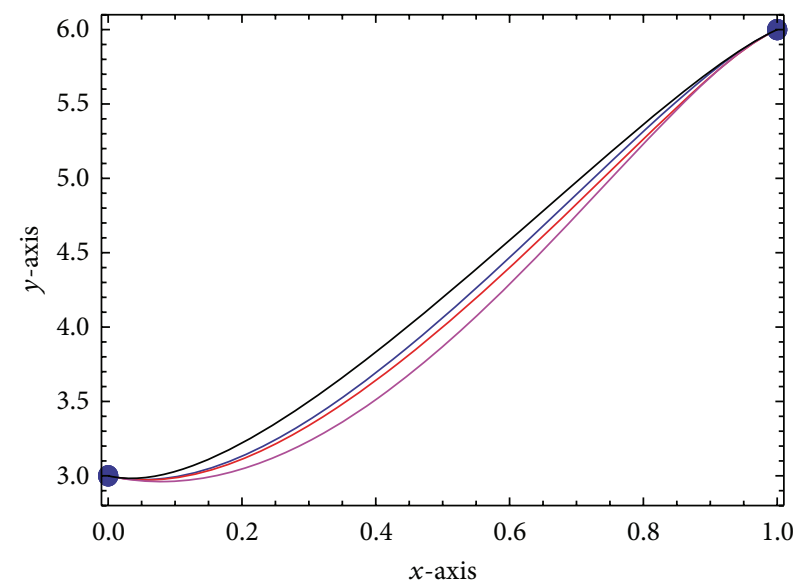

FIgURE 9: The graph of the rational interpolation curves $P_{1}(t)$ (blue), $P_{2}(t)$ (red), $P_{4}(t)$ (purple), and $P_{5}(t)$ (gray).

Now, if we assigned the value of $\alpha_{i}$ and $\beta_{i}$, for example, we choose $\beta_{i}=\gamma_{i}=1$, then (15) becomes

$$
P\left(\frac{1}{2}, \alpha_{i}\right)=\frac{5 \alpha_{i}+10+9+18 \alpha_{i}}{2\left(\alpha_{i}+1+1+2 \alpha_{i}\right)}=\frac{23 \alpha_{i}+19}{2\left(3 \alpha_{i}+2\right)} .
$$

Now, if we require $P(1 / 2)=N$, then

$$
\frac{23 \alpha_{i}+19}{2\left(3 \alpha_{i}+2\right)}=N \text {. }
$$

Equation (26) can be rearranged as follows:

$$
\alpha_{i}=\frac{19-4 N}{6 N-23} \text {. }
$$

Due to the fact that the parameter $\alpha_{i}>0$, thus (27) gives the following constraints to the value of $N$ when we choose $\beta_{i}=\gamma_{i}=1$ :

$$
\frac{23}{6}<N<\frac{19}{4} \quad \text { or } \quad N \in\left(3 \frac{5}{6}, 4.75\right) .
$$


Now, if we assigned the value of $\alpha_{i}$ and $\beta_{i}$, for example, we choose $\alpha_{i}=2$ and $\beta_{i}=1$, then (15) becomes

$$
P\left(\frac{1}{2}, \gamma_{i}\right)=\frac{10+10+9 \gamma_{i}+36}{2\left(2+1+\gamma_{i}+4\right)}=\frac{9 \gamma_{i}+56}{2\left(\gamma_{i}+7\right)} \text {. }
$$

Now, if we require $P(1 / 2)=N$, then

$$
\frac{9 \gamma_{i}+56}{2\left(\gamma_{i}+7\right)}=N
$$

Equation (30) can be rearranged as follows:

$$
\gamma_{i}=\frac{56-14 N}{2 N-9}
$$

Due to the fact that the parameter $\gamma_{i} \geq 0$, (31) gives the constraints to the value of $N$ when we choose $\alpha_{i}=2$ and $\beta_{i}=1,4 \leq N \leq 4.5$ or $N \in[4,4.5]$.

Similarly if we choose $\alpha_{i}=2$ and $\gamma_{i}=1$, then

$$
P\left(\frac{1}{2}, \beta_{i}\right)=\frac{10+10 \beta_{i}+9+36 \beta_{i}}{2\left(2+1+\beta_{i}+2 \beta_{i}\right)}=\frac{19 \beta_{i}+46}{6\left(\beta_{i}+1\right)} \text {. }
$$

By rearrange (32),

$$
\begin{aligned}
& \frac{19+46 \beta_{i}}{2\left(5 \beta_{i}+3\right)}=N . \\
& \beta_{i}=\frac{19-6 N}{10 N-46} .
\end{aligned}
$$

Due to the fact that the parameter $\beta_{i}>0$, (31) gives the following constraints to the value of $N$ when we choose $\alpha_{i}=2$ and $\gamma_{i}=1$ :

$$
3 \frac{1}{6} \leq N \leq 4.6 \quad \text { or } \quad N \in\left(3 \frac{1}{6}, 4.6\right)
$$

Thus we have three possible ranges for $N$ for the different choices of parameters $\alpha_{i}, \beta_{i}$, and $\gamma_{i}$. We may obtain different values of ranges of $N$ for the different values of the parameters. What we want to know is the range for $N$ to ensure the user or designer will choose the suitable values of $N$ satisfying $P\left(t^{*}\right)=N$; otherwise we may end in solving nonlinear equations without any possible solution in $t \in(0,1)$.

Remark 5. From (28) the following can be observed:

$$
\text { (a) } \lim _{\alpha_{i} \rightarrow 0} P\left(\frac{1}{2}, \alpha_{i}\right)=\frac{19}{4}, \quad \text { (b) } \lim _{\alpha_{i} \rightarrow \infty} P\left(\frac{1}{2}, \alpha_{i}\right)=\frac{23}{6} \text {. }
$$

\section{Inflection-Point and Convexity Control of Curves}

From calculus, the convexity and concavity of an interpolating curve depend on the second-order derivative of the interpolating function. The second derivative of the rational cubic spline with three parameters defined by (1) is given in (36) below [23]:

$$
P^{\prime \prime}(t)=\frac{\sum_{j=0}^{3} C_{i j}(1-\theta)^{3-j} \theta^{j}}{h_{i}\left[(1-\theta)^{2} \alpha_{i}+\theta(1-\theta)\left(2 \alpha_{i} \beta_{i}+\gamma_{i}\right)+\theta^{2} \beta_{i}\right]^{3}},
$$

where

$$
\begin{gathered}
C_{i 0}=2 \alpha_{i}^{2}\left(\gamma_{i}\left(\Delta_{i}-d_{i}\right)-\beta_{i}\left(d_{i+1}-\Delta_{i}\right)-2 \alpha_{i} \beta_{i}\left(d_{i}-\Delta_{i}\right)\right), \\
C_{i 1}=6 \alpha_{i}^{2} \beta_{i}\left(\Delta_{i}-d_{i}\right), \quad C_{i 2}=6 \alpha_{i} \beta_{i}^{2}\left(d_{i+1}-\Delta_{i}\right), \\
C_{i 3}=2 \beta_{i}^{2}\left[\gamma_{i}\left(d_{i+1}-\Delta_{i}\right)-\alpha_{i}\left(\Delta_{i}-d_{i}-2 \beta_{i}\left(d_{i+1}-\Delta_{i}\right)\right)\right] .
\end{gathered}
$$

Now let

$$
M(\theta)=\sum_{j=0}^{3} C_{i j}(1-\theta)^{3-j} \theta^{j}
$$

By simple calculation it can be shown that

$$
\begin{aligned}
& M(0)=2 \alpha_{i}^{2}\left(\gamma_{i}\left(\Delta_{i}-d_{i}\right)-\beta_{i}\left(d_{i+1}-\Delta_{i}\right)-2 \alpha_{i} \beta_{i}\left(d_{i}-\Delta_{i}\right)\right), \\
& M(1)=2 \beta_{i}^{2}\left[\gamma_{i}\left(d_{i+1}-\Delta_{i}\right)-\alpha_{i}\left(\Delta_{i}-d_{i}-2 \beta_{i}\left(d_{i+1}-\Delta_{i}\right)\right)\right] .
\end{aligned}
$$

If $M(0) M(1)<0$, the interpolation curve $P(t)$ must have inflection point which satisfies $P^{\prime \prime}(t)=0$ at $t=t^{*}$. Thus we have the following Theorem.

Theorem 6. Given the interpolating data $\left(t_{i}, f_{i}, d_{i}\right), i=$ $0,1, \ldots, n$, the interpolation function $P(t)$ defined by (1) in each subinterval $\left[t_{i}, t_{i+1}\right], i=0,1,2, \ldots, n-1$, has inflection point except when both $M(0)$ and $M(1)$ are equal to zero and $\left(6 \alpha_{i}^{3} \Delta_{i}-4 \alpha_{i}^{3} d_{i}-2 \alpha_{i}^{2} d_{i}\right) \times\left(4 d_{i+1}+2 \alpha_{i} d_{i+1}-6 \Delta_{i}\right)<0$ except for the case $d_{i+1}-\Delta_{i}=\Delta_{i}-d_{i}$.

From Duan et al. [18], the inflection point of the interpolating curve $P(t)$ can be controlled. If the design requires the inflection point to be at the point $\left(t^{*}, P\left(t^{*}\right)\right)$, then the positive parameters $\alpha_{i}, \beta_{i}$, and $\gamma_{i}$ can be selected from the following equation:

$$
M\left(\theta^{*}\right)=0
$$

where $M\left(\theta^{*}\right)$ is defined by (38). Similarly if the design requires the convex point (or concave point) to be at $\left(t^{*}, P\left(t^{*}\right)\right)$, then the positive parameters $\alpha_{i}, \beta_{i}$, and $\gamma_{i}$ must satisfy the following condition:

$M\left(\theta^{*}\right)>0 \quad($ for convex $)$ or $M\left(\theta^{*}\right)<0 \quad($ for concave $)$.

Thus, we have our final theorem of inflection-point and convexity (concavity) control of interpolating curves.

Theorem 7. Given the interpolating data $\left(t_{i}, f_{i}, d_{i}\right), i=$ $0,1, \ldots, n$, the interpolation function $P(t)$ defined by $(1)$ in each subinterval $\left[t_{i}, t_{i+1}\right], i=0,1,2, \ldots, n-1$, has inflection point 
TABLE 1: Interpolation data from Duan et al. [18].

\begin{tabular}{lc}
\hline$f_{i}$ & $d_{i}$ \\
\hline 3 & -1 \\
6 & 2 \\
\hline
\end{tabular}

at the point $\left(t^{*}, P\left(t^{*}\right)\right)$, if there exist positive parameters $\alpha_{i}, \beta_{i}$, and $\gamma_{i}$ satisfying (40), and has convex point (or concavity) at $\left(t^{*}, P\left(t^{*}\right)\right)$, if there exist positive parameters $\alpha_{i}, \beta_{i}$, and $\gamma_{i}$ that satisfy (41).

Remark 8. If the data gives $\Delta_{i}-d_{i}=0$ or $d_{i+1}-\Delta_{i}=0$, then we may set $d_{i}=d_{i+1}=\Delta_{i}$. This will result in the rational cubic interpolant in (1) reproducing the linear segment in that interval:

$$
s(x)=(1-\theta) f_{i}+\theta f_{i+1} .
$$

Example 9. Let $f(t)$ be the interpolated function for the interpolation data given in Table 1. From Example 4, when $\alpha_{i}=2, \beta_{i}=1$, and $\gamma_{i}=1$, the interpolating function $P(t)$ in the interval $[0,1]$ is given as

$$
P_{1}(t)=\frac{6+t+14 t^{2}-15 t^{3}}{2+t-2 t^{2}} .
$$

The second-order derivative of $P(t)$ is given by

$$
P^{\prime \prime}(t)=\frac{168-408 t+300 t^{2}-86 t^{3}}{\left(2+t-2 t^{2}\right)^{3}} .
$$

Simple calculation shows that $P^{\prime \prime}(0)=21$ and $P^{\prime \prime}(1)=-26$; thus for $t \in(0,1)$ there must exist inflection point for $P(t)$ given in (43) above. The root of the equation $P^{\prime \prime}(t)=0$ in $(0,1)$ is

$$
\begin{aligned}
t^{*}= & \frac{1}{43}\left[50-\frac{4 \sqrt[3]{106^{2}}}{(-157+43 \sqrt{7})^{1 / 3}}\right] \\
& +[106(-157+43 \sqrt{17})]^{1 / 3} \approx 0.6999144 .
\end{aligned}
$$

Thus when $0 \leq t \leq t^{*}$, the interpolating function $P(t)$ is convex and when $t^{*} \leq t \leq 1$, the interpolating function $P(t)$ is concave. These properties can be verified from the graph of $P^{\prime \prime}(t)$ shown in Figure 10.

Now if the design requires the inflection point to be at $t=0.6$, the parameters $\alpha_{i}, \beta_{i}$, and $\gamma_{i}$ must be selected to satisfy the requirement $M\left(0.6, \alpha_{i}, \beta_{i}, \gamma_{i}\right)=0$. Now from (41), with $t=0.6$, the following equation is obtained:

$$
\begin{aligned}
M\left(\alpha_{i}, \beta_{i}, \gamma_{i}\right)=\frac{2}{125}[ & 64 \alpha_{i}^{3} \beta_{i}-54 \alpha_{i} \beta_{i}^{2}\left(3+\beta_{i}\right) \\
& \left.-27 \beta_{i}^{2} \gamma_{i}+8 \alpha_{i}^{2}\left(19 \beta_{i}+4 \gamma_{i}\right)\right] .
\end{aligned}
$$

Let $\beta_{i}=1 / 2$ and $\gamma_{i}=1$; then $M\left(\alpha_{i}\right)$ becomes

$$
M\left(0.6, \alpha_{i}\right)=\frac{1}{250}\left[128 \alpha_{i}^{3}+432 \alpha_{i}^{2}-189 \alpha_{i}-27\right] .
$$

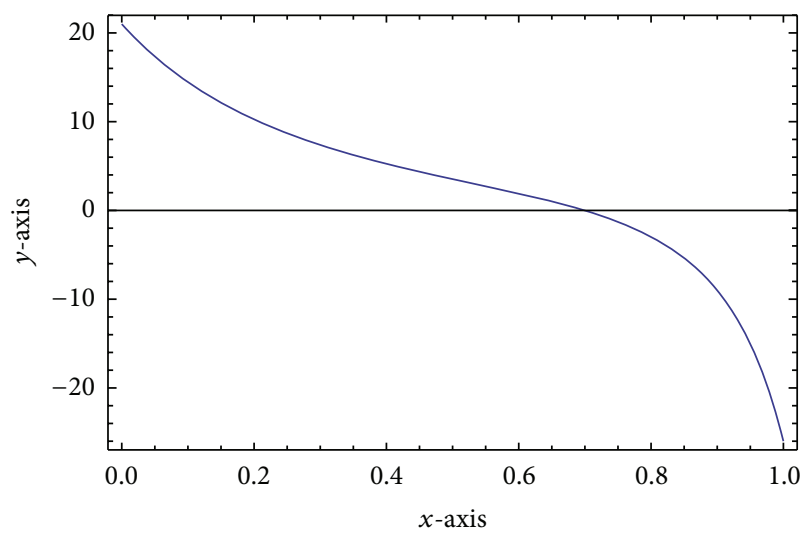

Figure 10: The graph of $P^{\prime \prime}(t)$.

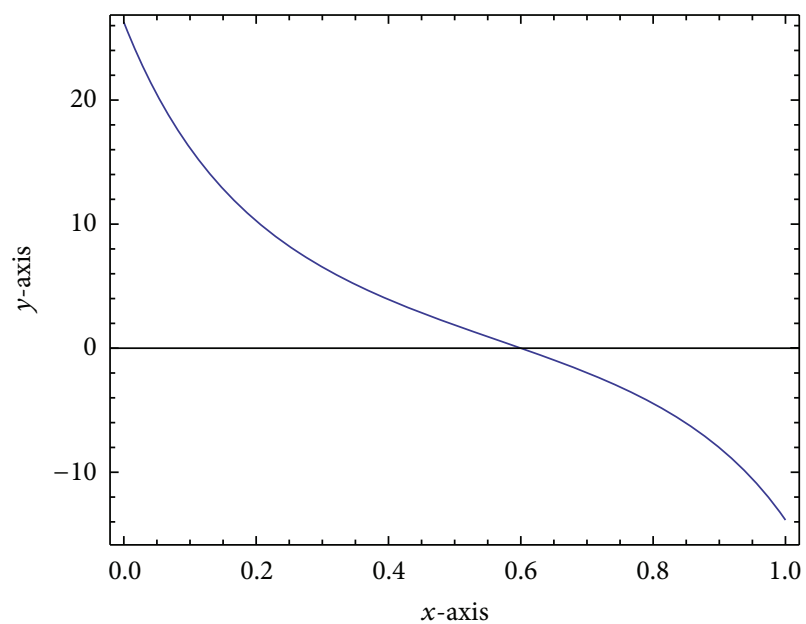

FIgURE 11: The graph of $P_{6}^{\prime \prime}(t)$.

The positive root on $t \in(0,1)$ of the equation $M\left(0.6, \alpha_{i}\right)=0$ is

$$
\begin{array}{r}
\alpha_{i}=\frac{3}{16}\left(-6+5 \sqrt{2} \cos \left[\frac{1}{3}\left(\arctan \left[\frac{\sqrt{4681}}{163}\right]\right)\right]\right. \\
\left.+5 \sqrt{6} \sin \left[\frac{1}{3}\left(\arctan \left[\frac{\sqrt{4681}}{163}\right]\right)\right]\right)
\end{array}
$$

$$
\approx 0.4925233 \text {. }
$$

Thus the interpolating function $P_{6}(t)$ after some simplification is given by

$$
P_{6}(t)=\frac{1.477568+1.029910 t+4.462613 t^{2}-3.970090 t^{3}}{0.492526+0.507477 t-0.5 t^{2}} .
$$

It can be seen clearly that from Figures 11 and 22 the $P_{6}^{\prime \prime}(t)$ has an inflection point at $t=0.6$. Figure 12 shows the graph of $P_{6}(t)$. Thus for the interpolating function $P_{6}(t)$, the graph is convex on $[0,0.6]$ and is concave on $[0.6,1]$ and the inflection point is $\left(0.6, P_{6}(0.6)\right)$. 


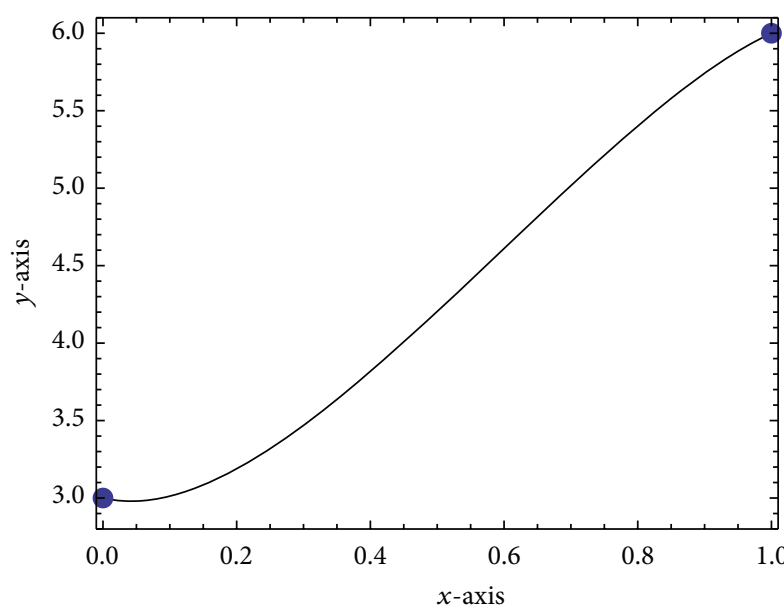

Figure 12: The graph of $P_{6}(t)$.

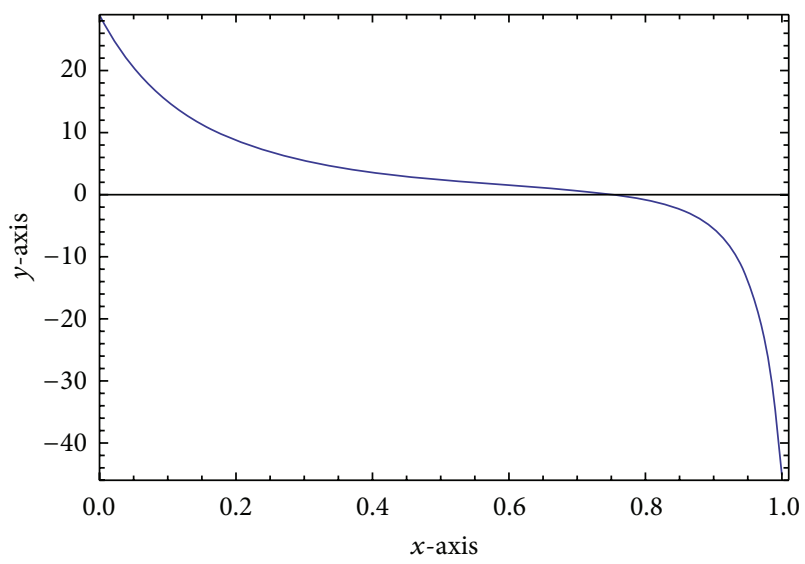

Figure 13: The graph of $P_{7}^{\prime \prime}(t)$.

Similarly, if the design requires the interpolating curve to be convex at $t=0.75$, then the suitable value of positive parameters $\alpha_{i}, \beta_{i}$, and $\gamma_{i}$ must be chosen from $M\left(0.75, \alpha_{i}, \beta_{i}, \gamma_{i}\right)>0$. Now $M\left(0.75, \alpha_{i}, \beta_{i}, \gamma_{i}\right)>0$ reduces to the following inequality:

$$
\begin{aligned}
M\left(\alpha_{i}, \beta_{i}, \gamma_{i}\right)=\frac{1}{32}[ & 8 \alpha_{i}^{3} \beta_{i}-27 \alpha_{i} \beta_{i}^{2}\left(5+2 \beta_{i}\right) \\
& \left.-27 \beta_{i}^{2} \gamma_{i}+\alpha_{i}^{2}\left(37 \beta_{i}+4 \gamma_{i}\right)\right]>0 .
\end{aligned}
$$

If we let $\alpha_{i}=3 / 10$ and $\beta_{i}=1 / 10$, the inequality in (50) becomes

$$
M\left(\gamma_{i}\right)=\frac{9}{16000}\left[-37+50 \gamma_{i}\right]>0 .
$$

The solution for (50) is $\gamma_{i}>37 / 50$. Thus the interpolating function $P_{7}(t)$ when $\alpha_{i}=3 / 10, \beta_{i}=1 / 10$, and $\gamma_{i}=1$ is given by

$$
P_{7}(t)=\frac{45+54 t+95 t^{2}-164 t^{3}}{15+23 t-33 t^{2}} .
$$

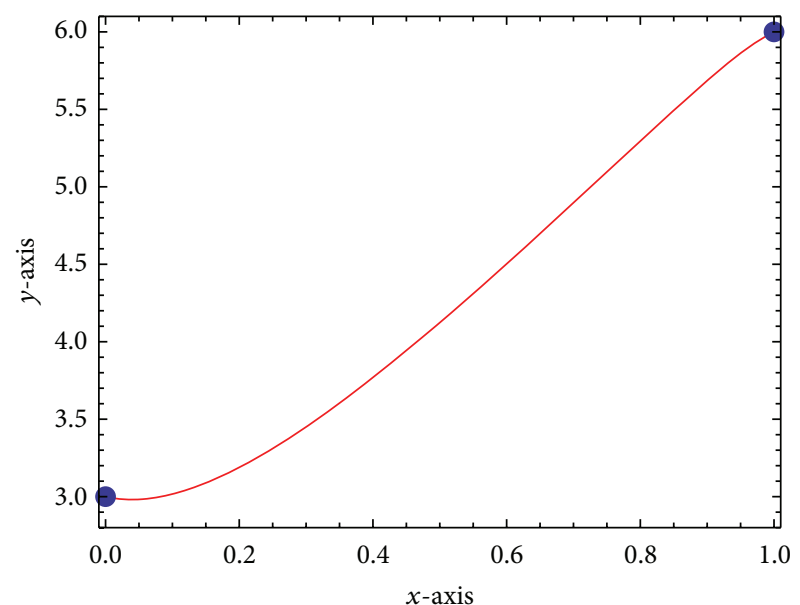

Figure 14: The graph of $P_{7}(t)$.

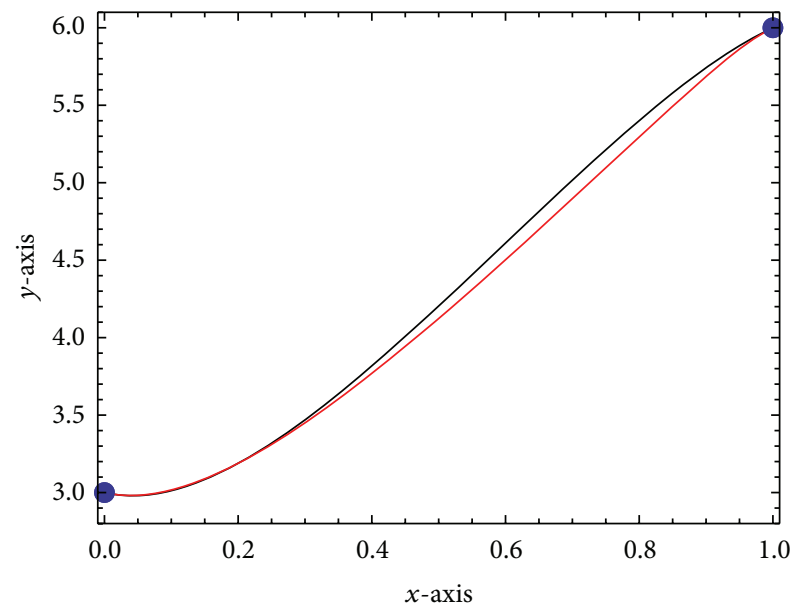

Figure 15: The graph of $P_{6}(t)$ (black) and $P_{7}(t)$ (red).

It can be verified that the interpolating curve $P_{7}(t)$ is convex at $t=0.75$ (Figure 21). The graph of $P_{7}^{\prime \prime}(t)$ shows that the interpolating curve is convex on $[0,0.75]$ and concave on $[0.75,1]$ (Figure 23). Figure 13 shows the graph of $P_{7}^{\prime \prime}(t)$. Meanwhile Figure 14 shows the graph of interpolating function $P_{7}(t)$. Finally Figures 15 and 16 show the comparison between the graphs of $P_{1}(t), P_{6}(t)$, and $P_{7}(t)$, respectively.

This subsection discusses the comparison between the local control interpolation by using our proposed rational cubic spline with three parameters and the work of Duan et al. [18]. We still use the same data given in Table 1. From Duan et al. [18], the interpolating function $P_{8}(t)$ is concave at $t=0.6$ when $\alpha_{i}=1 / 2$ and $\beta_{i}=(19+\sqrt{2521}) / 135 \approx 0.512663$. The interpolating function $P_{8}(t)$ is given as follows:

$$
\begin{aligned}
P_{8}(t)= & \left((4 \sqrt{2521}-1679) t^{3}+(8 \sqrt{2521}+827) t^{2}\right. \\
& +675 t+405) \\
\times & \left(135+270 t+(2 \sqrt{2521}-367) t^{2}\right)^{-1} .
\end{aligned}
$$




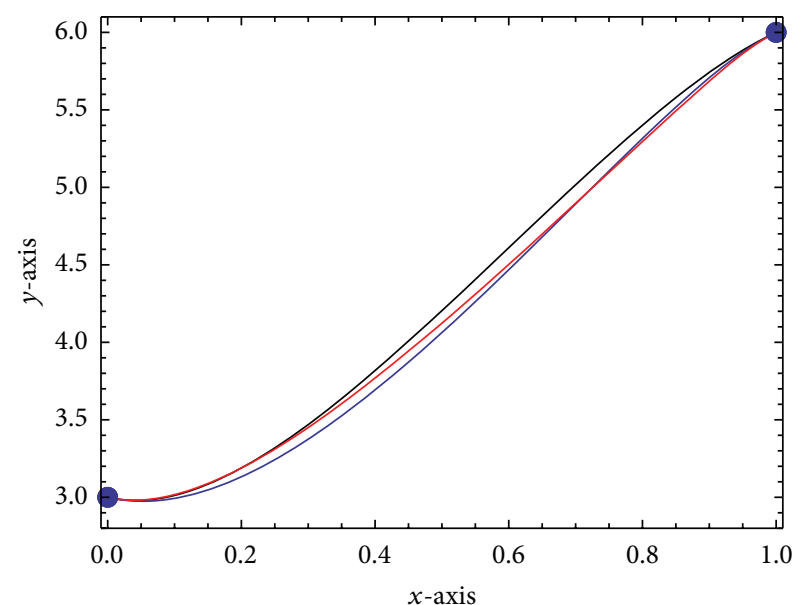

Figure 16: The graph of $P_{1}(t)$ (blue), $P_{6}(t)$ (black), and $P_{7}(t)$ (red).

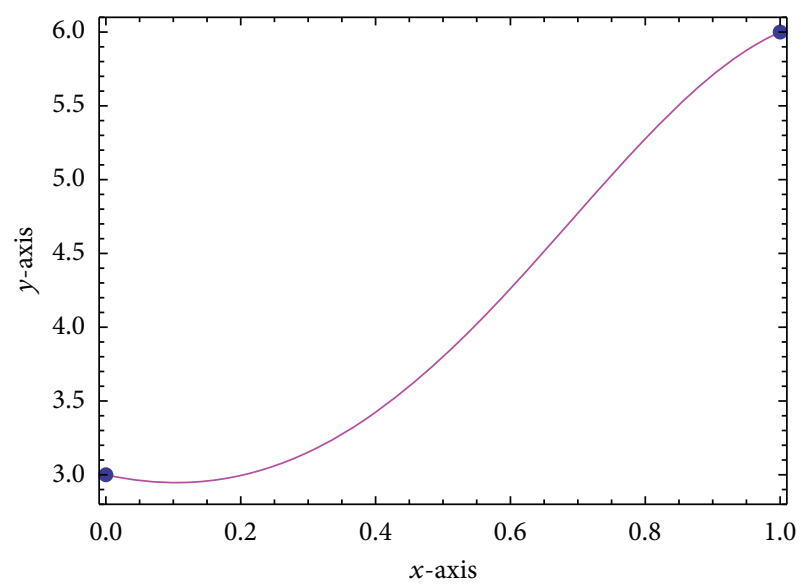

FIgURE 17: The graph of interpolating function of Duan et al. [18].

Similarly the rational interpolating function $P_{9}(t)$ is convex at $t=0.75$ when $\alpha_{i}=1$ and $0 \leq \beta_{i} \leq(37+\sqrt{7417}) / 378$ (Figure 21). The interpolating function $P_{9}(t)$ with $\alpha_{i}=1$ and $\beta_{i}=0.3$ is given as follows:

$$
P_{9}(t)=\frac{2\left(32 t^{3}-31 t^{2}+5 t-15\right)}{7 t^{2}-10} .
$$

Figures 17 and 18 show the graph of the interpolating functions $P_{8}(t)$ and $P_{9}(t)$, respectively. Meanwhile Figures 19 and 20 show the comparison between the concave-point control and local convex control by using our proposed rational cubic spline and the work of Duan et al. [18]. By careful inspection to Figures 12,14, 17, and 18, it can be seen clearly that the local control by using the proposed rational cubic spline with three parameters gives comparable results with the established work by Duan et al. [18].

Remark 10. If we choose two positive values such as $\beta_{i}$ and $\gamma_{i}$, then (50) will be cubic functions and any symbolic software can be used to solve the equation. By having three parameters,

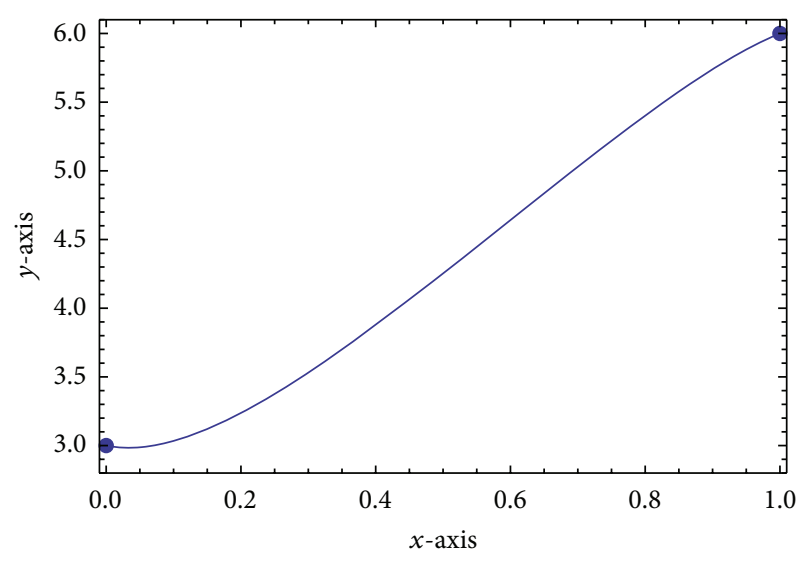

Figure 18: The graph of $P_{8}(t)$.

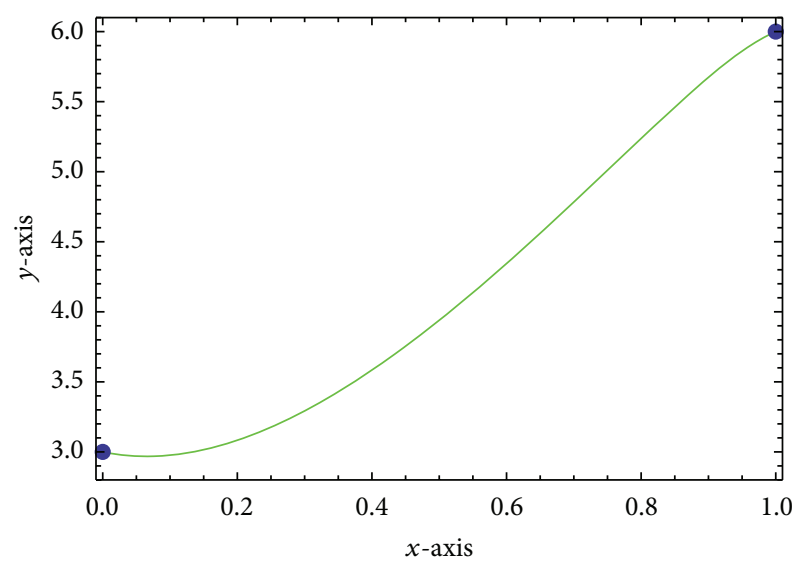

Figure 19: The graph of $P_{9}(t)$.

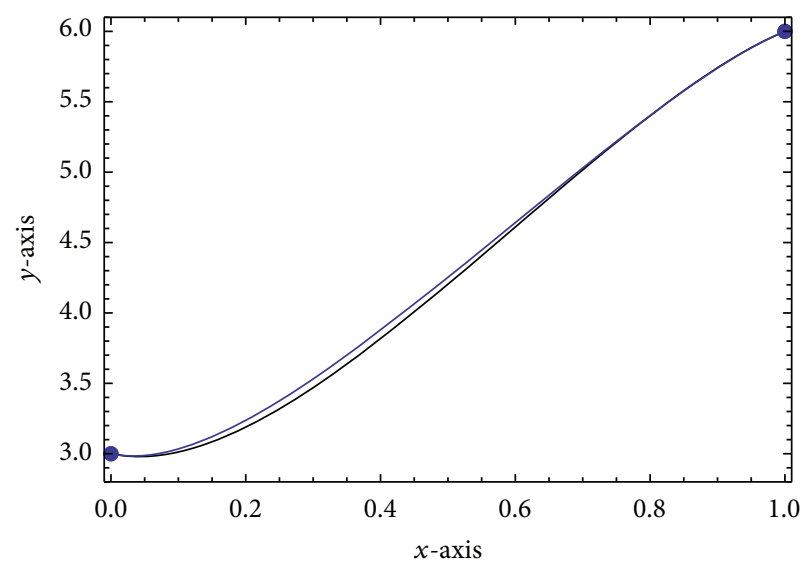

Figure 20: The graph of $P_{6}(t)$ (black) and $P_{8}(t)$ (blue).

we avoid any nonlinear equation from (50). In Duan et al. [18], to obtain the interpolating function $P_{8}(t)$ in (51), we need to solve the quadratic equation. Furthermore the convexity preserving (for convex data) by using our rational cubic spline with three parameters can be obtained in [23]. 


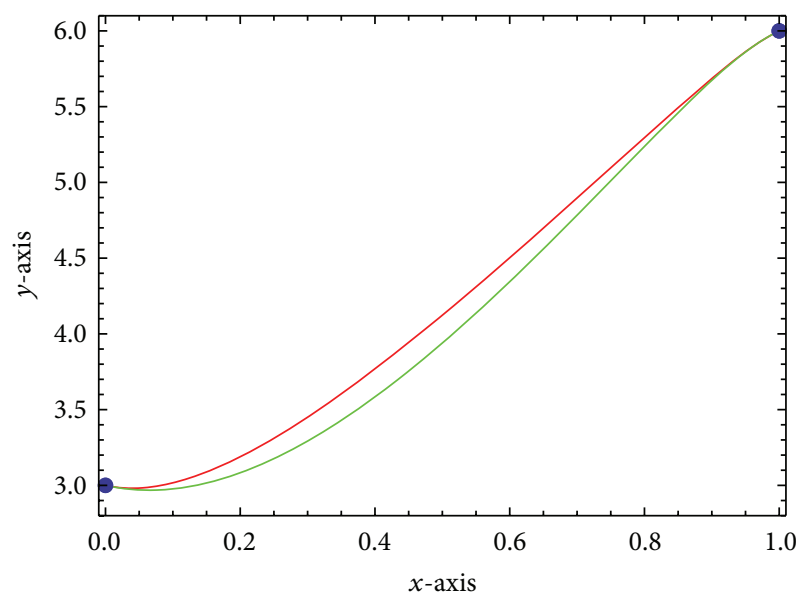

FIgURE 21: The graph of $P_{7}(t)\left(\right.$ red) and $P_{9}(t)$ (green).

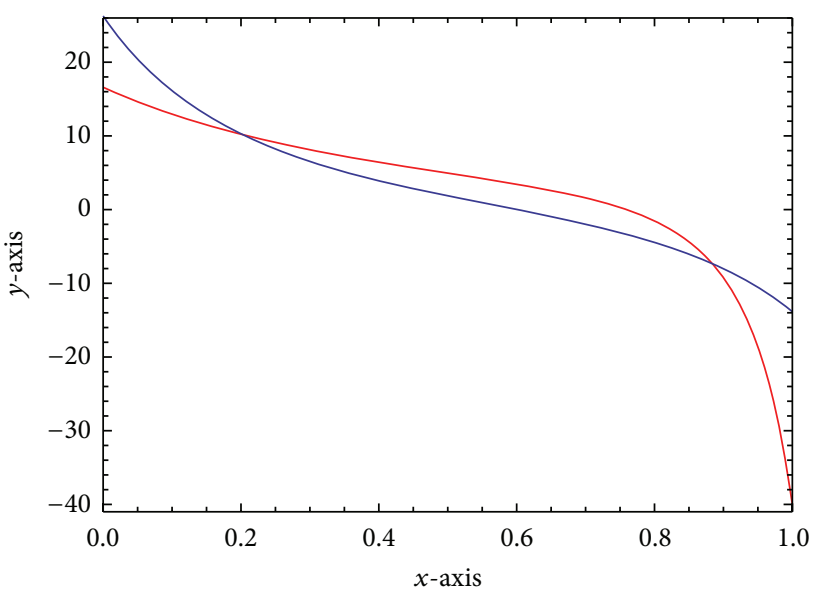

Figure 22: The graph of $P_{6}^{\prime \prime}(t)$ (blue) and $P_{8}^{\prime \prime}(t)$ (red).

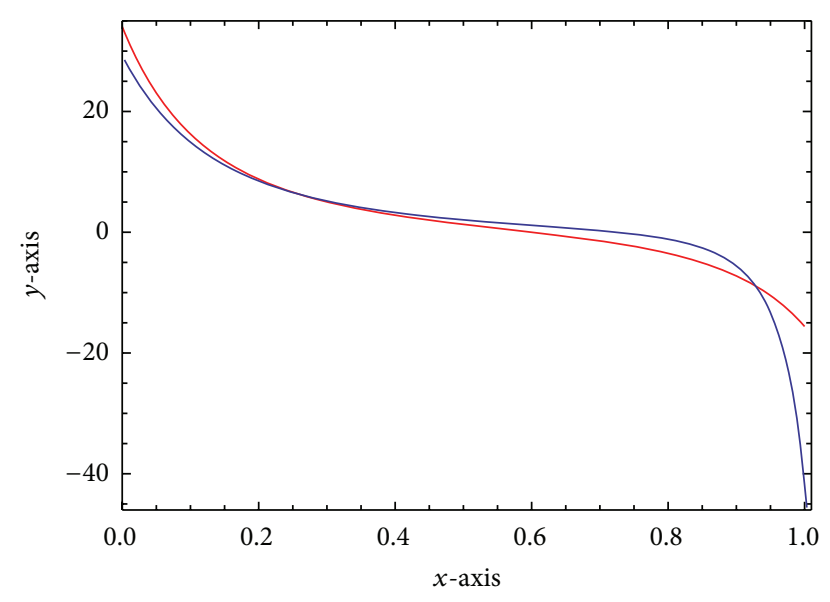

FIgURE 23: The graph of $P_{7}^{\prime \prime}(t)$ (blue) and $P_{9}^{\prime \prime}(t)$ (red).

\section{Conclusions}

The new rational cubic spline with the three parameters proposed by Karim and Kong [21-23] has been used for local control of interpolation data. The parameters in the description of the rational interpolant have been selected for the local control of interpolating curves, namely, point-value control, inflection, and convexity control, without the need to change the interpolation data points. The results indicated that, by having more parameters, the local control can be achieved with a greater flexibility and quite more easiness. Since, we have two parameters to choose, the final parameter is calculated from Theorems 1, 3, 6, and 7, respectively. Comparisons between the proposed methods and the work of Duan et al. [18] also have been made. From careful inspection to all presented graphs, our rational scheme works well and it is comparable with the work of Duan et al. [18]. Furthermore, this work can be extended for the bounded interpolation on surface by using the same idea from the work of Duan et al. [25]. Work on local control by using function values with our rational cubic spline is underway. This will be our main subject for future research.

\section{Conflict of Interests}

The authors declare that there is no conflict of interests regarding the publication of this paper.

\section{Acknowledgments}

The first author would like to acknowledge Universiti Teknologi PETRONAS for the financial support received in the form of a research grant: Short Term Internal Research Funding (STIRF) no. 35/2012. Comments and suggestions from the referees have improved this paper significantly.

\section{References}

[1] S. Butt and K. W. Brodlie, "Preserving positivity using piecewise cubic interpolation," Computers \& Graphics, vol. 17, no. 1, pp. 5564, 1993.

[2] K. W. Brodlie and S. Butt, "Preserving convexity using piecewise cubic interpolation," Computers \& Graphics, vol. 15, no. 1, pp. 1523, 1991.

[3] R. L. Dougherty, A. S. Edelman, and J. M. Hyman, "Nonnegativity-, monotonicity-, or convexity-preserving cubic and quintic Hermite interpolation," Mathematics of Computation, vol. 52, no. 186, pp. 471-494, 1989.

[4] F. N. Fritsch and R. E. Carlson, "Monotone piecewise cubic interpolation," SIAM Journal on Numerical Analysis, vol. 17, no. 2, pp. 238-246, 1980.

[5] F. N. Fritsch and J. Butland, "A method for constructing local monotone piecewise cubic interpolants," SIAM Journal on Scientific and Statistical Computing, vol. 5, no. 2, pp. 300-304, 1984.

[6] L. L. Schumaker, "On shape preserving quadratic spline interpolation," SIAM Journal on Numerical Analysis, vol. 20, no. 4, pp. 854-864, 1983.

[7] J. W. Schmidt and W. Hess, "Positivity of cubic polynomials on intervals and positive spline interpolation," BIT, vol. 28, no. 2, pp. 340-352, 1988.

[8] M. Sarfraz, "Visualization of positive and convex data by a rational cubic spline interpolation," Information Sciences, vol. 146, no. 1-4, pp. 239-254, 2002.

[9] M. Sarfraz, M. Z. Hussain, and A. Nisar, "Positive data modeling using spline function," Applied Mathematics and Computation, vol. 216, no. 7, pp. 2036-2049, 2010. 
[10] M. Z. Hussain, M. Sarfraz, and T. S. Shaikh, "Shape preserving rational cubic spline for positive and convex data," Egyptian Informatics Journal, vol. 12, no. 3, pp. 231-236, 2011.

[11] T. S. Shaikh, M. Sarfraz, and M. Z. Hussain, "Shape preserving constrained data visualization using rational functions," Journal of Prime Research in Mathematics, vol. 7, pp. 35-51, 2011.

[12] M. Tian, Y. Zhang, J. Zhu, and Q. Duan, "Convexity-preserving piecewise rational cubic interpolation," Journal of Information and Computational Science, vol. 2, no. 4, pp. 799-803, 2005.

[13] F. Bao, Q. Sun, and Q. Duan, "Point control of the interpolating curve with a rational cubic spline," Journal of Visual Communication and Image Representation, vol. 20, no. 4, pp. 275-280, 2009.

[14] F. Bao, Q. Sun, J. Pan, and Q. Duan, "Point control of rational interpolating curves using parameters," Mathematical and Computer Modelling, vol. 52, no. 1-2, pp. 143-151, 2010.

[15] Q. Duan, F. Bao, S. Du, and E. H. Twizell, "Local control of interpolating rational cubic spline curves," Computer Aided Design, vol. 41, no. 11, pp. 825-829, 2009.

[16] F. Bao, Q. Sun, J. Pan, and Q. Duan, "A blending interpolator with value control and minimal strain energy," Computers \& Graphics, vol. 34, no. 2, pp. 119-124, 2010.

[17] Q. Sun, F. Bao, J. Pan, and Q. Duan, "Reconstruction of curves with minimal energy using a blending interpolator," Mathematical Methods in the Applied Sciences, vol. 36, no. 10, pp. 1301-1309, 2013.

[18] Q. Duan, X. Liu, and F. Bao, "Local shape control of the rational interpolation curves with quadratic denominator," International Journal of Computer Mathematics, vol. 87, no. 1-3, pp. 541-551, 2010.

[19] J. A. Gregory, M. Sarfraz, and P. K. Yuen, "Interactive curve design using $C^{2}$ rational splines," Computers \& Graphics, vol. 18, no. 2, pp. 153-159, 1994.

[20] J. Pan and Q. Sun, "Shape control of the curves using rational cubic spline," Journal of Computational Information Systems, vol. 9, pp. 5861-5868, 2013.

[21] S. A. A. Karim and V. P. Kong, "Shape preserving interpolation using rational cubic spline," to be published in Research Journal of Applied Sciences, Engineering and Technology, 2014.

[22] S. A. A. Karim and V. P. Kong, "Monotonicity-preserving using rational cubic spline interpolation," to be published in Research Journal of Applied Sciences, 2014.

[23] S. A. A. Karim and V. P. Kong, "Convexity-preserving using rational cubic spline interpolation," to be published in Research Journal of Applied Sciences, Engineering and Technology, 2014.

[24] U. Bashir and J. M. Ali, "Data visualization using rational trigonometric spline," Journal of Applied Mathematics, vol. 2013, Article ID 531497, 10 pages, 2013.

[25] Q. Duan, H. Zhang, Y. Zhang, and E. H. Twizell, "Bounded property and point control of a bivariate rational interpolating surface," Computers \& Mathematics with Applications, vol. 52, no. 6-7, pp. 975-984, 2006. 


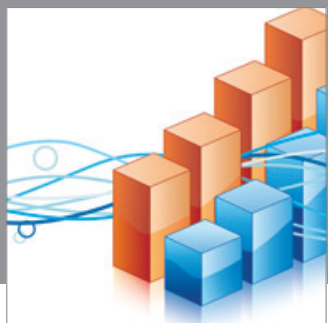

Advances in

Operations Research

mansans

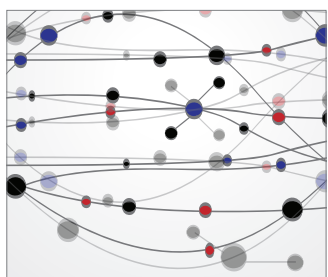

The Scientific World Journal
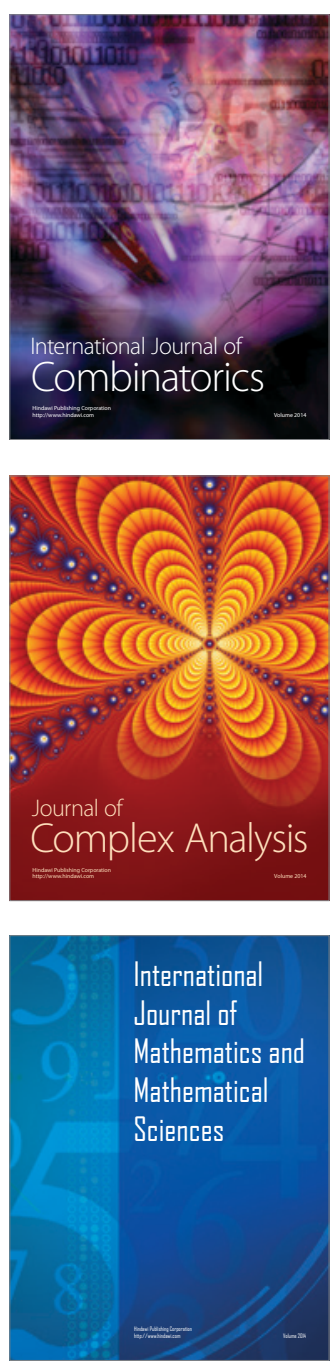
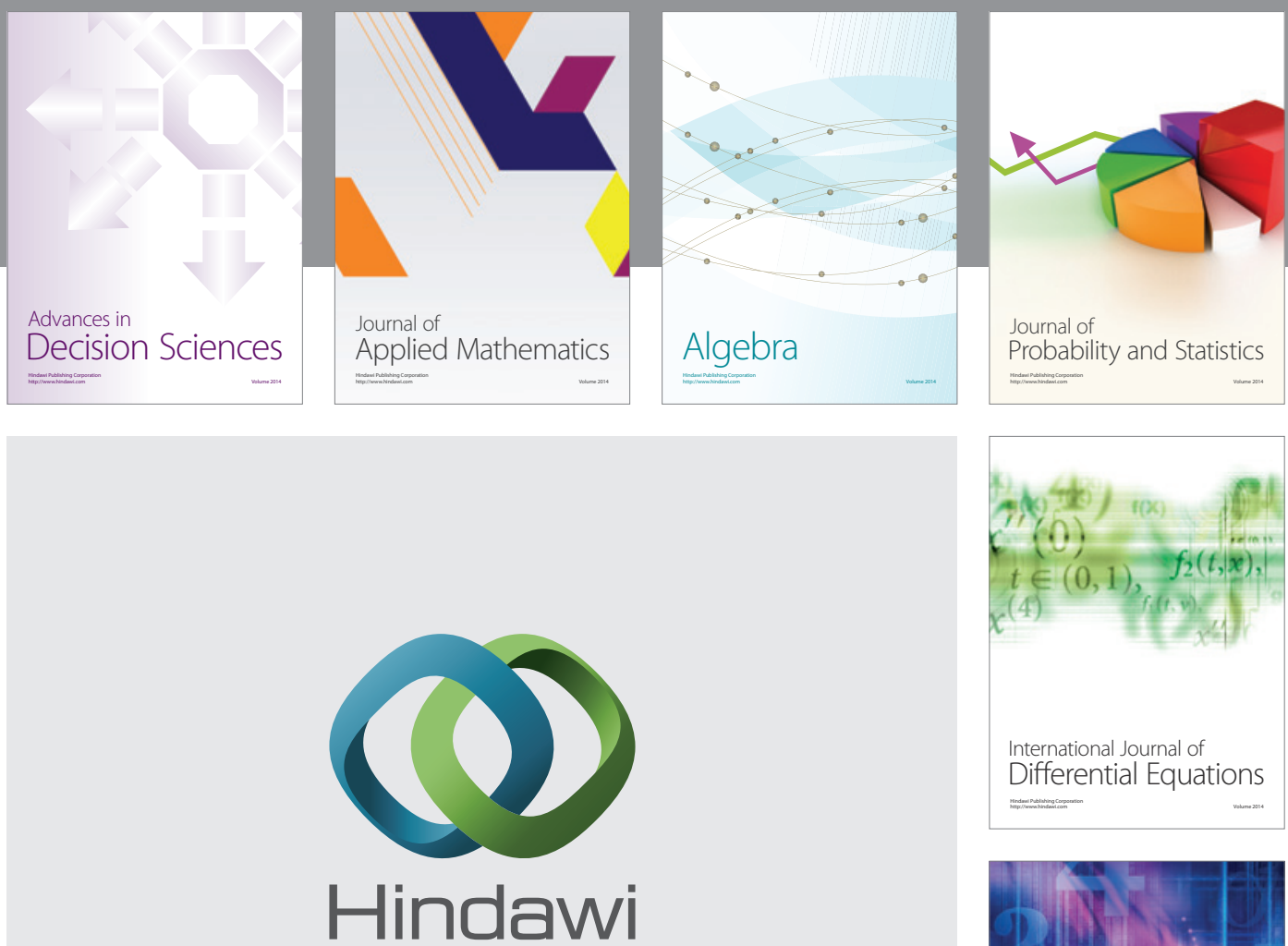

Submit your manuscripts at http://www.hindawi.com
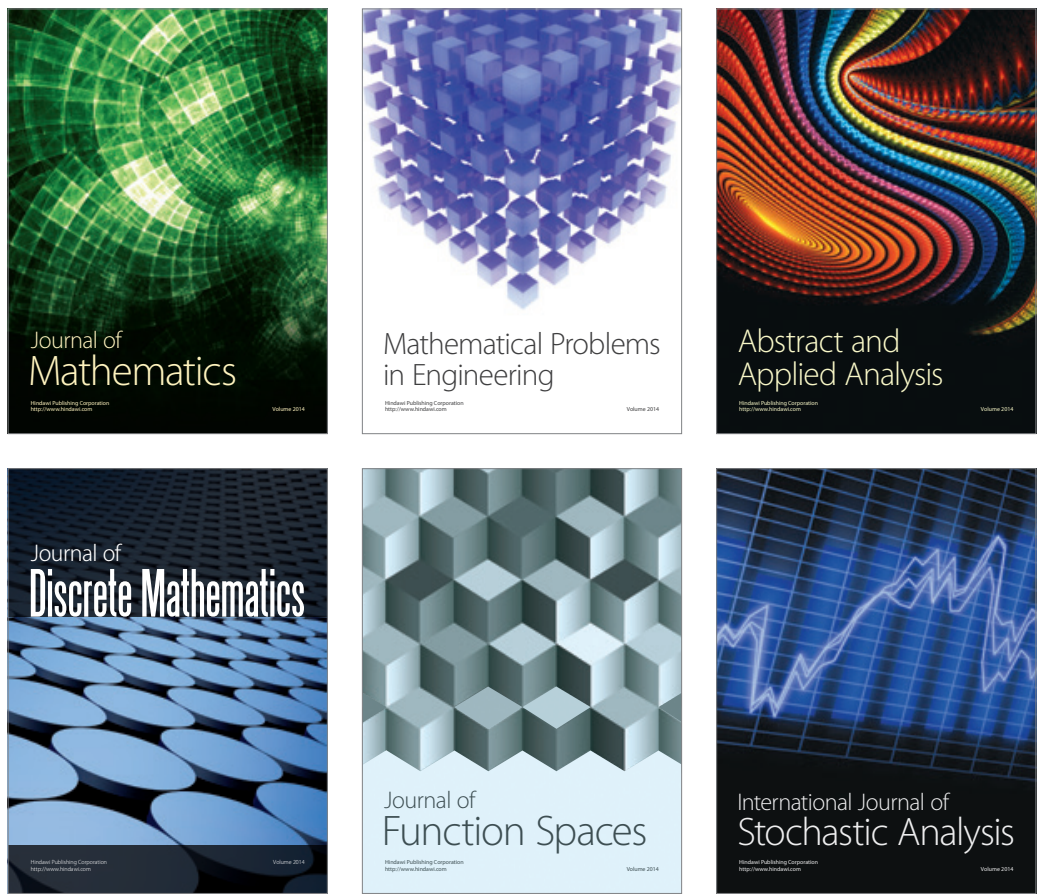

Journal of

Function Spaces

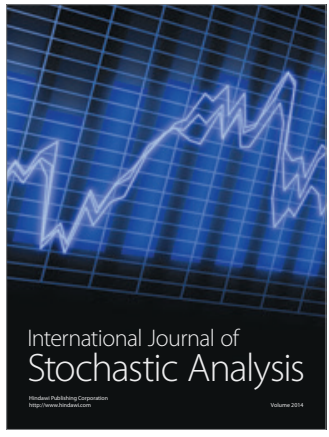

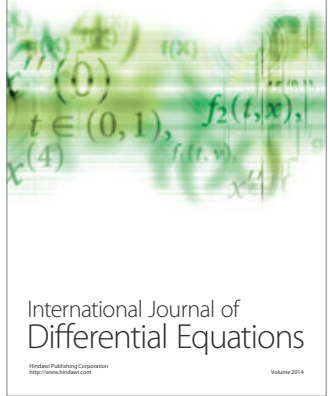
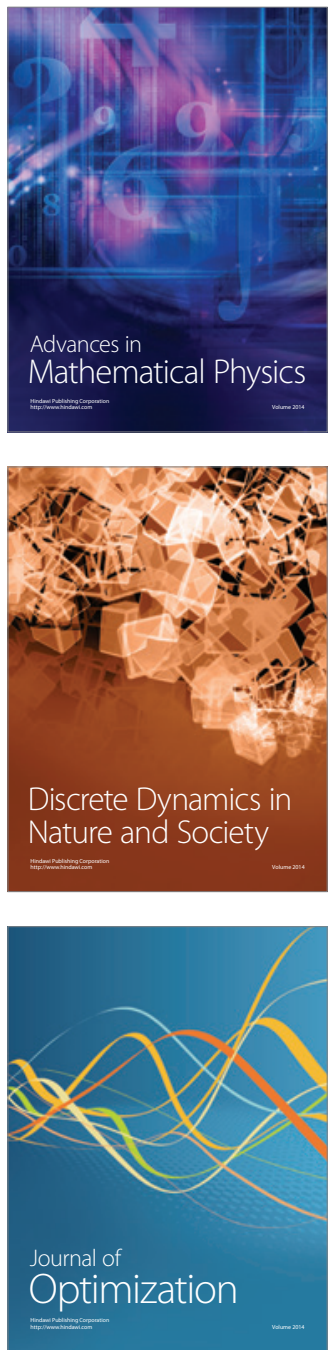